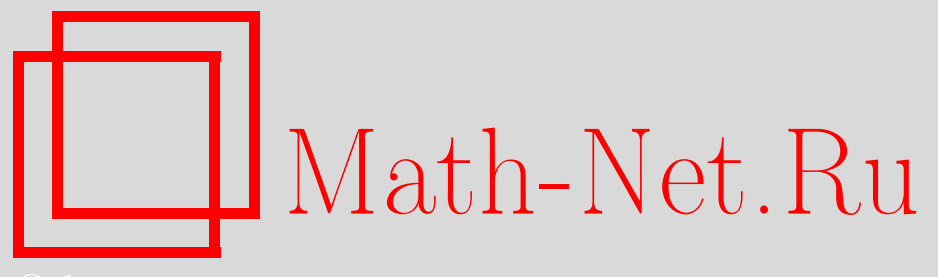

В. Г. Михайлов, А. М. Шойтов, Структурная эквивалентность $s$-цепочек в случайных дискретных последовательностях, Дискрет. матем., 2003, том 15, выпуск 4, 7-34

DOI: https://doi.org/10.4213/dm214

Использование Общероссийского математического портала Math-Net.Ru подразумевает, что вы прочитали и согласны с пользовательским соглашением http://www.mathnet.ru/rus/agreement

Параметры загрузки:

IP : 54.224 .135 .184

26 апреля 2023 г., $17: 37: 21$

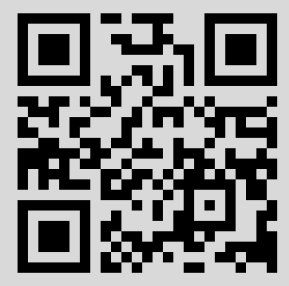




\title{
Аискретная математика
}

том 15 выпуск $4 * 2003$

УдК 519.2

\section{Структурная эквивалентность $s$-цепочек в случайных дискретных последовательностях}

\author{
() 2003 г. В. Г. Михайлов, А. М. Шойтов
}

\begin{abstract}
В последние годы было опубликовано значительное число работ о структурных свойствах случайных дискретных последовательностей. В них была представлена большая группа результатов о структурно эквивалентных отрезках в таких последовательностях. Нами предлагается обзор постановок задач и полученных решений в этом интересном направлении дискретной теории вероятностей.

Работа поддержана Российским фондом фундаментальных исследований, грант 02-01-00266, грантом НШ 1758.2003.1 Президента Российской Федерации для поддержки ведущих научных школ Российской Федерации, а также грантом МК 2831.2003.09 Президента Российской Федерации для поддержки научных исследований молодых ученых - кандидатов наук.
\end{abstract}

\section{1. Введение}

Пусть $\left(a_{1}, \ldots, a_{s}\right),\left(b_{1}, \ldots, b_{s}\right)$ - цепочки букв некоторого конечного алфавита $A$. Под структурой цепочки мы понимаем расположение одинаковых и разных букв в ней. Другими словами, две цепочки $\left(a_{1}, \ldots, a_{s}\right)$ и $\left(b_{1}, \ldots, b_{s}\right)$ обладают одинаковой структурой в том и только том случае, когда

$$
a_{i}=a_{j} \Longleftrightarrow b_{i}=b_{j}, \quad 1 \leqslant i<j \leqslant s .
$$

Это условие эквивалентно условию

$$
a_{i} \neq a_{j} \Longleftrightarrow b_{i} \neq b_{j}, \quad 1 \leqslant i<j \leqslant s .
$$

Цепочки $\left(a_{1}, \ldots, a_{s}\right)$ и $\left(b_{1}, \ldots, b_{s}\right)$ называются эквивалентными, если существует такая подстановка $h$ на множестве букв алфавита $A$, при которой

$$
b_{i}=h\left(a_{i}\right), \quad i=1, \ldots, s .
$$

Очевидно, что эквивалентными являются цепочки с одинаковой структурой и только они.

Существует понятие, относительно которого совпадение цепочек и совпадение лишь их структур являются частными случаями. Это понятие $H$-связанности или $H$-совпадения цепочек. Рассмотрим множество всех подстановок элементов алфавита $A$. Оно является группой относительно умножения подстановок и обозначается $S(A)$ или $S_{N}$, где $N=|A|$. Пусть $H$ - некоторое подмножество множества $S(A)$. 
Цепочки $\left(a_{1}, \ldots, a_{s}\right)$ и $\left(b_{1}, \ldots, b_{s}\right)$ назовем $H$-связанными (и будем говорить об $H$-совпадения этих цепочек и писать $\left.\left(a_{1}, \ldots, a_{s}\right) H\left(b_{1}, \ldots, b_{s}\right)\right)$, если

$$
\exists h \in H ; \quad b_{i}=h\left(a_{i}\right), \quad i=1, \ldots, s .
$$

Если множество подстановок $H$ является подгруппой групшы $S(A)$, то отношение, определяемое на множестве $A^{s}$ соотношением (3), является отношением эквивалентности. В этом случае $\boldsymbol{H}$-связанные цепочки принято называть $\boldsymbol{H}$-эквивалентными (см. [2]).

Понятия эквивалентности и $S(A)$-эквивалентности цепочек совпадают. При $H=\{e\}$ (то есть в случае, когда $H$ - группа, состоящая из одной тождественной подстановки $e$ ) $H$-эквивалентность означает совпадение цепочек.

Если $H_{1} \subseteq H_{2} \subseteq S(A)$, то $H_{1}$-связанные цепочки являются и $H_{2}$-связанными. Отсюда следует, что $H$-связанные цепочки обладают одинаковой структурой.

Наличие в дискретной последовательности большого числа цепочек с одинаковой структурой заметно снижает ее сложность и позволяет добиваться большего сжатия данных при архивации (см., например, $[11,12,58])$. Важную роль при оценке эффективности процедуры сжатия играют величины $\xi_{r}(H)$, число $r$-кратных $H$-повторений $s$-цепочек в отрезке, и, если множество $H$ является группой подстановок, величины $\mu_{r}(H)$, число классов $H$-эквивалентных $s$-цепочек, представители которых встретились в отрезке последовательности ровно $r$ раз.

Типичная постановка задачи для $\xi_{r}(H)$ имеет следующий вид. Пусть

$$
X=\left(X_{1}, X_{2}, \ldots, X_{n+s-1}\right)
$$

- отрезок последовательности независимых одинаково распределенных случайных величин, принимающих значения в множестве $A=\{1,2, \ldots, N\}$ с вероятностями $p_{1}, \ldots, p_{N}$ соответственно. Введем обозначения

$$
\begin{aligned}
E_{i j}(h) & =\left\{\left(h\left(X_{i}\right), \ldots, h\left(X_{i+s-1}\right)\right)=\left(X_{j}, \ldots, X_{j+s-1}\right)\right\} \\
E_{i j}(H) & =\left\{\left(X_{i}, \ldots, X_{i+s-1}\right) H\left(X_{j}, \ldots, X_{j+s-1}\right)\right\}=\bigcup_{h \in H} E_{i j}(h) .
\end{aligned}
$$

Каждой паре $(i, j), 1 \leqslant i<j \leqslant n$, сопоставим случайную величину

$$
W_{i j}(H)=I\left(E_{i j}(H)\right)
$$

(здесь и далее выражение $I(\cdot)$ обозначает индикатор случайного события или неслучайного множества, указанного в скобках). Тогда число $r$-кратных наборов $\boldsymbol{H}$-связанных $s$-цепочек среди $n$ первых $s$-цепочек последовательности $X$ выразится формулой

$$
\xi_{r}(H)=\sum_{1 \leqslant i_{1}<\ldots<i_{r} \leqslant n} W_{i_{1}, i_{2}}(H) \ldots W_{i_{r-1}, i_{r}}(H) .
$$

Выписать распределение случайной величины $\xi_{r}(H)$ в сколь-либо общем случае представляется нереальным. Поэтому основное внимание исследователей было привлечено к нахождению предельных распределений случайных величин $\xi_{r}(H)$ при $n \rightarrow \infty$ и согласованно с $n$ меняющихся параметрах $s$ и $p_{1}, \ldots, p_{N}$.

Полученные результаты об асимптотических свойствах случайных величин $\xi_{r}(H)$ и $\mu_{r}(H)$ можно условно разделить на три группы. Первую группу образуют предельные теоремы для $\xi_{r}(H)$ при малых множествах $H$. Теоремы пуассоновского типа в данном 
случае являются непосредственными обобщениями аналогичных предельных теорем для числа повторений $s$-цепочек в последовательности независимых испытаний, полученных А. М. Зубковым и В. Г. Михайловым [15] еще в 1974 году. Здесь в качестве $H$ выступают одноточечные множества и групты типа латинского прямоугольника. Сюда же относится ситуация, когда при переходе к пределу алфавит и распределение букв остаются фиксированными. В последнем случае возможно любопытное явление, названное редукцией, когда значения $\xi_{r}(H)$ (здесь множество $H$-группа) в пределе совпадают с величиной $\xi_{r}\left(H_{\mathscr{P}}\right)$, где подгруппа $H_{\mathscr{P}}$ группы $H$ может быть много меньше $H$ и даже состоять из одного элемента. В случае фиксированного распределения букв были описаны и условия сходимости распределения $\xi_{r}(H)$ к нормальному распределению и к распределению типа хи-квадрат. Изложению этих результатов посвящен раздел 2.

Вторую группу составляют предельные теоремы пуассоновского типа для случайных величин $\xi_{r}(H)$ при больших множествах $H$. Все они являются, в конечном счете, обобщениями полученной С. М. Буравлевым в девяностые годы предельной теоремы Пуассона для числа пар цепочек с одинаковой структурой в последовательности независимых равновероятных знаков. Работа [9], посвященная доказательству этой теоремы, по сути дела дала старт разработке данного научного направления. Любопытной особенностью поведения случайных величин $\xi_{r}(H)$ при больших множествах $H$ является возможная сильная зависимость $\xi_{r}\left(H_{1}\right)$ и $\xi_{r}\left(H_{2}\right)$ даже при непересекающихся множествах $H_{1}$ и $H_{2}$. Изложению этих результатов посвящен третий раздел обзора.

Третью группу составляют результаты, полученные путем сведения задачи об $H$-эквивалентных цепочках к задачам о конечно-зависимых размещениях частиц по ячейкам. Частным случаем последней является задача о числе $\mu_{r}(H)$ классов $H$-эквивалентных $s$-цепочек, представители которых встретились в отрезке последовательности ровно $r$ раз. Эти результаты изложены в разделе 4.

Ряд результатов, приведенных в обзоре, не укладывается в эту условную схему. Это задачи об $H$-связанных цепочках из двух или более последовательностей и вопросы об иных формах эквивалентности цепочек. Им посвящены разделы 5 и 6 соответственно.

Следует заметить, что обзор содержит ряд новых результатов, доказательства которых будут опубликованы в ближайшее время.

\section{2. Предельные теоремы при малых множествах $H$}

Изложение результатов о $H$-эквивалентных и $H$-связанных цепочках мы начнем с предельной теоремы для числа пар одинаковых цепочек (в наших обозначениях это $\xi_{2}(H)$ при $H=\{e\})$ в последовательности независимых испытаний, доказанной А. М. Зубковым и В. Г. Михайловым в 1974 году [15]. Эта теорема оказала заметное влияние на первоначальное развитие данного научного направления.

Введем обозначения. Пусть $X_{1}, \ldots, X_{n+s-1}$ - отрезок последовательности независимых одинаково распределенных случайных величин, принимающих значения из алфавита $A=\{1,2, \ldots, N\}$,

$$
\begin{gathered}
\mathbf{P}\left\{X_{i}=k\right\}=p_{k}, \quad k=1, \ldots, N, \quad \mathscr{P}=\left(p_{1}, \ldots, p_{N}\right), \quad p=\max _{1 \leqslant k \leqslant N} p_{k}, \\
P=n p^{s}, \quad \sum_{k=1}^{N} p_{k}^{r}=P_{r}, \quad r=2,3, \ldots
\end{gathered}
$$

Здесь $s$ - длина рассматриваемых цепочек. 
Теорема 1 ([15]). Пусть $n \rightarrow \infty$, а параметры $s, N$ и вероятности $p_{1}, \ldots, p_{N}$ меняются так, что

$$
\begin{gathered}
\left(\begin{array}{l}
n \\
2
\end{array}\right)\left(1-P_{2}\right) P_{2}^{s} \rightarrow \lambda, \quad 0<\lambda<\infty, \\
P_{2} \rightarrow \rho \in[0,1), \quad s^{t} n p^{s} \rightarrow 0
\end{gathered}
$$

для любого фиксированного неотричательного $t$.

Тогда распределение случайной величины $\xi_{2}(\{e\})$ сходится к сложному пуассоновскому распределению с производящей функчией

$$
\varphi_{\lambda, \rho}(z)=\exp \left\{\frac{\lambda(z-1)}{1-\rho z}\right\} .
$$

Замечание 1. Если $\rho>0$, то при переходе к пределу совпадения цепочек образуют группы, образование такой группы представляет собой редкое событие, их число имеет в пределе пуассоновское распределение. Количество пар совпавших цепочек в такой группе имеет в пределе геометрическое распределение. Производящая функция $\varphi_{\lambda, \rho}(z)$ отвечает распределению суммы пуассоновского с параметром $\lambda$ числа независимых случайных величин, каждая из которых имеет геометрическое распределение с параметром $\rho$. При $\rho=0$ предельное распределение в теореме 15 оказывается просто пуассоновским.

Этот результат неоднократно обобщался и уточнялся. В [21] он был перенесен на случайные величины $\xi_{r}=\xi_{r}(\{e\})$, в [17] была получена оценка скорости сходимости в этой предельной теореме. Современные методы позволяют заменить условие $s^{t} n p^{s} \rightarrow 0$ на более слабое условие $s n p^{s} \rightarrow 0$.

Обращаясь к доказательствам теорем в $[15,21]$, нетрудно убедиться, что утверждение теоремы 1 справедливо не только для $\xi_{2}(\{e\})$, но и при любом одноточечном множестве $H$ и любых $r \geqslant 2$. При этом формулировка теоремы практически не меняется.

Пусть

$$
P_{r}(h)=\sum_{k=1}^{N} p_{k} p_{h(k)} \ldots p_{h^{r-1}(k)},
$$

где $h$ - некоторая подстановка на алфавите $A, h^{m}(k)=h\left(h^{m-1}(k)\right), m=2, \ldots, r-1$.

Теорема 2. IІусть $n \rightarrow \infty, h-$ некоторая подстановка на алфавите $A, r \geqslant 2-$-заданное натуральное число, а параметры $s, N$ и вероятности $p_{1}, \ldots, p_{N}$ меняются так, что

$$
\begin{gathered}
\left(\begin{array}{l}
n \\
r
\end{array}\right)\left(1-P_{r}(h)\right) P_{r}^{s}(h) \rightarrow \lambda, \quad 0<\lambda<\infty, \\
P_{r}(h) \rightarrow \delta \in[0,1), \quad s^{t} n p^{s} \rightarrow 0
\end{gathered}
$$

для любого фиксированного неотрицательного $t$.

Тогда распределение случайной величины $\xi_{r}(\{h\})$ сходится к сложному пуассоновскому распределению с производящей функцией $\varphi_{\lambda, \delta}(z)$.

В работах $[6-10,38]$ исследовалось число $\xi_{2}(H)$ пар $H$-эквивалентных $s$-цепочек в случае, когда множество $H$ является набором $\left\{h_{1}, \ldots, h_{k}\right\}$ из $k$ подстановок, для которых строки $\left(h_{i}(1), \ldots, h_{i}(N)\right), i=1, \ldots, k$ (будучи выписаны одна под другой) образуют латинский прямоугольник. В этом случае $H$ является группой подстановок, которую называют группой типа латинского прямоугольника или просто латинским прямоугольником.

Из теоремы 2 работы [10] вытекает следующее утверждение. 
Теорема 3. Пусть $n \rightarrow \infty$, а параметры $k, s, N, p_{1}, \ldots, p_{N}$ и множество $H$ меняются так, что нижние строки подстановок из $Н$ образуют латинский прямоугольник, $s^{q} n p^{s} \rightarrow 0$ для любого фиксированного неотричательного $q, p \leqslant c<1, p^{2} \leqslant P_{2}\left(h_{t}\right)$, $t=1, \ldots, m, P_{2}\left(h_{t}\right) \rightarrow \delta_{t} \in[0,1)$ равномерно по $t=1, \ldots, k$, подстановки в множестве н упорядочены так, что $\delta=\delta_{1}=\ldots=\delta_{m}>\delta_{m+1} \geqslant \ldots \geqslant \delta_{k}$ при некотором $1 \leqslant m \leqslant k$ $u$

$$
\left(\begin{array}{l}
n \\
2
\end{array}\right)\left(P\left(h_{t}\right)\right)^{s}\left(1-P\left(h_{t}\right)\right) \rightarrow \lambda_{t}, \quad 0<\lambda_{t}<\infty, \quad 1 \leqslant m \leqslant k .
$$

Тогда для любых фиксированных $z<\delta^{-1} u k=1,2, \ldots$

$$
E z^{\xi_{2}(H)}=\prod_{t=1}^{m} \exp \left\{\frac{\lambda_{t}(z-1)}{1-\delta z}\right\}(1+o(1)) .
$$

Теорема 3 дает пример ситуащии, в которой влияние на предельное распределение может оказывать лишь часть группы $H$. Это явление мы будем называть редукщией статистики $\xi_{2}(H)$. Более подробно оно было изучено А. М. Шойтовым в постановке, когда при переходе к пределу алфавит и распределение букв на нем остаются неизменными. Им доказано, что при определенных условиях предельное распределение $\xi_{r}(H)$ определяется не самой группой $H$, а ее подгруппой

$$
H_{\mathscr{P}}=\left\{h \in H: p_{a}=p_{h(a)}, a=1, \ldots, N\right\},
$$

и указаны достаточные условия сходимости к сложному пуассоновскому распределению такому, как и в теореме 1. Статья с изложением этих результатов сдана в печать.

Теорема 4. Пусть параметры $N$ и $r$ и вероятности $p_{a}>0, a=1, \ldots, N$, фиксированы, a параметры $n$ и s стремятся к бесконечности так, что

$$
n P_{r}^{s / r}=O(1)
$$

Тогда

$$
\lim _{n \rightarrow \infty} \mathbf{P}\left\{\xi_{r}(H)=\xi_{r}\left(H_{\mathscr{P}}\right)\right\}=1 .
$$

Особый интерес теорема 4 представляет в случае, когда $H_{\mathscr{\rho}}=\{e\}$. Он имеет место, например, если все вероятности $p_{1}, \ldots, p_{N}$ различны. Тогда из теорем 4 и 2 получаем следующее утверждение.

Следствие 1. Пусть группа $H$ и распределение $\mathscr{P}$ таковы, что $H_{\mathscr{P}}=\{e\}, r \geqslant 2-$ заданное натуральное число, а параметры $s, n$ стремятся к бесконечности так, что

$$
\left(\begin{array}{l}
n \\
r
\end{array}\right)\left(1-P_{r}\right) P_{r}^{s} \rightarrow \lambda, \quad 0<\lambda<\infty,
$$

и выполнено условие (5).

Тогда распределение случайной величины $\xi_{r}(H)$ сходится к сложному пуассоновскому распределению с производящей функчией $\varphi_{\lambda, \rho}(z)$.

Теорема 5. Пусть параметры $N, r$ и вероятности $p_{a}>0, a=1, \ldots, N$, фиксированы, a параметры n и s стремятся к бесконечности так, что

$$
\left(\begin{array}{l}
n \\
r
\end{array}\right) P_{r}^{s}\left(1-P_{r}\right)\left|H_{\Phi}\right|^{r-1} \rightarrow \lambda, \quad 0<\lambda<\infty .
$$


Тогда

$$
\lim _{n \rightarrow \infty} E z^{\xi_{r}(H)}=\exp \left\{\frac{\lambda(z-1)}{1-P_{r} z}\right\}
$$

Замечание 2. Оценки скорости сходимости в теореме 5 были приведены в [41].

Интересным является случай $p_{1}=\ldots=p_{N}=1 / N$. Из теоремы 5 вытекает следующее утверждение.

Следствие 2. Пусть схема равновероятна, то есть $p_{i}=1 / N, i=1, \ldots, N$, причем $N$, $r$ фиксированы, а параметры $n$ и s стремятся к бесконечности так, что

$$
\left(1-N^{-1}\right)\left(\begin{array}{l}
n \\
r
\end{array}\right)\left(\frac{|H|}{N^{s}}\right)^{r-1} \rightarrow \lambda, \quad 0<\lambda<\infty .
$$

Тогда

$$
E z^{\xi_{r}(H)} \rightarrow \exp \left\{\frac{\lambda(z-1)}{1-N^{1-r} z}\right\}
$$

Для случая $r=2$ этот результат был получен А. М. Шойтовым в [36].

Очевидно, что при любом множестве $H$ выполнено неравенство

$$
\xi_{2}(H) \leqslant \sum_{h \in H} \xi_{2}(\{h\})
$$

Из теоремы 5 можно вывести следующее утверждение.

Следствие 3. Пусть параметры $N, r$ и вероятности $p_{a}>0, a=1, \ldots, N$, фиксированы, а параметры п и стремятся к бесконечности так, что

$$
n^{r} P_{r}^{s}=O(1)
$$

Тогда

$$
\mathbf{P}\left\{\xi_{2}(H)=\sum_{h \in H_{i p}} \xi_{2}(\{h\})\right\} \rightarrow 1
$$

Замечание 3. Соотношение (8) не связано с видом предельного распределения в теореме 5 , а объясняется тем, что в рассматриваемой ситуации $H$-связанные цепочки достаточно длинны и каждая из них наверняка содержит все $N$ знаков алфавита. Поэтому любая пара $H$-связанных цепочек однозначно определяет связывающую подстановку $h \in H$.

Наряду с пуассоновским распределением в качестве предельного может выступать и нормальное распределение. В частности, это имеет место в постановке теоремы 5, если вместо (7) предположить, что $n^{r}\left(P_{r}\right)^{s} \rightarrow \infty$ достаточно медленно. Один такой вариант достаточных условий асимптотической нормальности был предложен А. М. Шойтовым.

Далее был изучен случай, когда при переходе к пределу неизменными остаются не только алфавит и распределение букв, но и длина рассматриваемых цепочек. Здесь можно воспользоваться тем, что задача о числе пар $H$-эквивалентных цепочек является частным случаем задачи о повторениях в последовательности конечно-зависимых случайных величин. Аналогичный подход в задаче о повторениях цепочек (то есть при $H=\{e\}$ ) был использован в работах $[22,23]$. 
Любое отношение эквивалентности $\phi$ на множестве $A^{s}$ слов длины $s$ над конечным алфавитом $A$ ( $s$-цепочек) может быть выражено с помощью некоторой функции $F_{\phi}: A^{s} \rightarrow Q$, где $Q$ - конечное множество, представляющее некоторой своей частью совокупность классов эквивалентности отношения $\phi$. Цепочки $\left(a_{1}, \ldots, a_{s}\right),\left(b_{1}, \ldots, b_{s}\right) \in A^{s}$ будут эквивалентными тогда и только тогда, когда

$$
F_{\phi}\left(a_{1}, \ldots, a_{s}\right)=F_{\phi}\left(b_{1}, \ldots, b_{s}\right) .
$$

Функция $F_{\phi}$ и множество $Q$ могут быть выбраны разными способами, лишь бы для эквивалентных цепочек (и только для них) выполнялось соотношение (9).

Эта интерпретация позволяет свести задачу об $H$-эквивалентных цепочках в последовательности независимых случайных величин $X_{1}, X_{2}, \ldots$ к задаче о повторениях для $(s-1)$-зависимых случайных величин

$$
Y_{i}=F\left(X_{i}, \ldots, X_{i+s-1}\right)
$$

при специально подобранной функции $F$.

В [38] было рассмотрено асимптотическое поведение числа наборов по $r$ попарно $H$-эквивалентных цепочек в ситуации, когда растет лишь длина последовательности, в которой наблюдаются цепочки. Оказалось, что в этом случае в качестве предельного распределения для $\xi_{r}(H)$ могут выступать только нормальное распределение и распределение типа хи-квадрат, причем тип предельного распределения определяется значениями вероятностей классов $H$-эквивалентности.

Пусть $\mathrm{M}_{s}[H]-$ множество классов $H$-эквивалентности множества $A^{s}$, класс эквивалентности с представителем $\bar{a}$ будем обозначать $K(\bar{a})$. Для любого класса $K$ из $\mathrm{M}_{s}[H]$ положим

$$
p(K)=\mathbf{P}\left(\left(X_{1}, \ldots, X_{s}\right) \in K\right)
$$

и определим множество

$$
N_{\mathscr{P}}(H)=\left\{s \in\{2,3, \ldots\}: p(K)=\left|\mathrm{M}_{s}[H]\right|^{-1}, \forall X \in \mathrm{M}_{s}[H]\right\}
$$

натуральных чисел $s \geqslant 2$, для которых все классы $\boldsymbol{H}$-эквивалентных $s$-цепочек имеют одинаковые вероятности. В [40] установлено, что $\xi_{r}(H)$ имеет в пределе нормальное распределение для всех $s \notin N_{\mathscr{P}}(H)$ и распределение типа хи-квадрат для всех $s \in N_{\mathscr{P}}(H)$. В частности, справедливы две следующие теоремы.

Пусть

$$
\begin{aligned}
\pi_{s}^{(r)}(H, \mathscr{P})= & \sum_{K \in \mathrm{M}_{s}[H]} p^{r}(K), \\
\Pi_{s, k}^{(r)}(H, \mathscr{P})= & \sum_{\left(a_{1}, \ldots, a_{s+k}\right) \in A^{k+s}} p_{a_{1}} \ldots p_{a_{k+s}} p^{r-1}\left(K\left(a_{1}, \ldots, a_{s}\right)\right) \\
& \times p^{r-1}\left(K\left(a_{1+k}, \ldots, a_{s+k}\right)\right), \quad k=0,1, \ldots, s-1, \\
\Pi_{s}^{(r)}(H, \mathscr{P})= & 2 \sum_{k=1}^{s-1} \Pi_{s, k}^{(r)}(H, \mathscr{P})+\Pi_{s, 0}^{(r)}(H, \mathscr{P})-(2 s-1)\left(\pi_{s}^{(r)}(H, \mathscr{P})\right)^{2} .
\end{aligned}
$$

Теорема 6. Если $s \notin N_{\mathscr{P}}(H)$, то при $n \rightarrow \infty$ распределение случайной величины

$$
\left(\Pi_{s}^{(r)}(H, \mathscr{P})\right)^{-1 / 2}(r-1) ! n^{1 / 2-r}\left(\xi_{r}(H)-\left(\begin{array}{l}
n \\
r
\end{array}\right) \pi_{s}^{(r)}(H, \mathscr{P})\right)
$$

сходится к стандартному нормальному закону. 
В [40] также получены условия равновероятности классов $\boldsymbol{H}$-эквивалентности. В частности, если $p_{a} \neq 1 / N$ для некоторого $a$ из $A$ или $H$ не является группой типа латинского прямоугольника, то множество $N_{\mathscr{P}}(H)$ содержит конечное множество натуральных чисел, причем если выполнено ровно одно их приведенных условий, то $N_{\mathscr{P}}(H)=\varnothing$. Отметим, что если выполнены сразу оба приведенные выше условия, то для $H=S_{N}$ всегда можно подобрать вектор вероятностей $\mathscr{P}$ так, что по крайней мере $2 \in N_{\mathscr{P}}(H)$. Если же $p_{a}=1 / N$ для всех $a$ из $A$ и $H$ - группа типа латинского прямоугольника, то $N_{\mathscr{P}}(H)$ состоит из всех натуральных чисел $s \geqslant 2$, в этом случае $\xi_{r}(H)$ имеет в пределе распределение типа хи-квадрат для всех $s \in N_{\mathscr{P}}(H)$, вид этого распределения приведен в следующей теореме.

Пусть

$$
\xi_{r}^{\prime}(H)=\left(N^{s}|H|\right)^{r-1} \xi_{r}(H), \quad \Delta_{m} z_{m}=z_{m}-z_{m-1}, \quad \Delta_{m}^{2} z_{m}=z_{m}-2 z_{m-1}+z_{m-2} .
$$

Теорема 7. Если $p_{a}=1 / N$ для всех а из $A$ и $H$ - группа типа латинского прямоугольника, то

- при $n \rightarrow \infty$ случайные величины $2(r-2) ! n^{1-r} \Delta_{s}^{2} \xi_{r}^{\prime}(H)+N^{s-2}(N-1)^{2} /|H|, s \geqslant 3$, асимптотически независимы и распределены по закону хи-квадрат с числом степеней свободы $N^{s-2}(N-1)^{2} /|H|$;

- при $n \rightarrow \infty$ случайные величины $2(r-2) ! n^{1-r} \Delta_{s} \xi_{r}^{\prime}(H)+N^{s-1}(N-1) /|H|, s \geqslant 2$, асимптотически распределены по закону хи-квадрат с $N^{s-1}(N-1) /|H|$ степенями свободы;

- если $|H| \geqslant 2$ и $s=2$, то при $n \rightarrow \infty$ случайная величина $2(r-2) ! n^{1-r} \xi_{r}^{\prime}(H)-$ $2 n /(r(r-1))+N^{2} /|H|$ в пределе распределена как сумма $Z_{1}^{2}+\left(1-3(|H| / N)^{2}\right) Z_{2}^{2}+$ $\left(1+2|H| / N-3(|H| / N)^{2}\right) Z_{3}^{2}$, где случайные величины $Z_{1}^{2}, Z_{2}^{2}$ и $Z_{3}^{2}$ независимы $и$ распределены по закону хи-квадрат с $(N /|H|)^{2}(|H|-1),(N /|H|)^{2}-N /|H| u N /|H|$ степенями свободы соответственно.

Здесь следует обратить внимание на то, что для группы типа латинского прямоугольника величина $|H|$ делит $N$ нацело.

\section{3. Предельные теоремы при больших множествах $H$}

Первый результат здесь был анонсирован С. М. Буравлевым в 1995 г. в тезисах [4] и касался асимптотических свойсгв числа $\xi_{2}\left(S_{N}\right)$ пар эквивалентных (то есть имеющих одинаковую структуру) $s$-цепочек в отрезке $X_{1}, \ldots, X_{n+s-1}$ рассматриваемой случайной последовательности. Этот результат положил начало исследованиям случайных величин $\xi_{r}(H)$ для нетривиальных групп подстановок $H$.

Теорема 8 ([4, 9]). Пусть случайные величины $X_{i}, i=1, \ldots, n+s-1$, независимы в совокупности, распределены на множестве А равновероятно и

$$
s N^{-1} \geqslant \ln N+1 \text {. }
$$

Тогда выполняется неравенство

$$
\lambda(s, n)=\frac{\left(\begin{array}{c}
n \\
2
\end{array}\right)}{N^{2 s}} \sum_{m=1}^{N} \sigma(s, m)\left((N)_{m}\right)^{2}<\left(\begin{array}{l}
n \\
2
\end{array}\right) \frac{N !}{N^{s}},
$$


где $\sigma(s, m)$ - числа Стирлинга второго рода; если $n, N, s \rightarrow \infty$ так, что $\lambda(s, n) \rightarrow \lambda>0$, mo

$$
\mathbf{P}\left\{\xi_{2}\left(S_{N}\right)=k\right\} \rightarrow \frac{\lambda^{k}}{k !} e^{-\lambda}, \quad k=0,1, \ldots
$$

при этом, если $s N^{-1}-\ln N \rightarrow \infty$, то

$$
\lambda(s, n)=\left(\begin{array}{l}
n \\
2
\end{array}\right) N ! N^{-s}(1+o(1)) .
$$

Подробное доказательство теоремы 8 приведено в [9], где были также получены вытекающие из нее предельные теоремы для случайных величин

$$
\tau(s)=\min \left\{n: \xi_{2}\left(S_{N}\right)>0\right\}, \quad \theta(n)=\max \left\{s: \xi_{2}\left(S_{N}\right)>0\right\} .
$$

В качестве предельных в них выступают те же самые распределения, что и в задаче о повторениях $s$-цепочек.

За последние годы было предпринято немало попыток обобщения теоремы 8 и ослабления ее условий. На этом пути были получены все приводимые ниже результаты этого раздела.

Естественным направлением исследований стало распространение теоремы 8 на другие группы. Это удалось сделать А. М. Шойтову в [36], где в качестве множества подстановок $H$ была взята произвольная подгруппа симметрической группы $S_{N}$. Основной результат, приведенный в [36], может быть сформулирован следующим образом.

Теорема 9. Пусть схема равновероятна: $p_{i}=1 / N, i=1, \ldots, N$, a параметры $n, N$ u $s$ стремятся к бесконечности так, что

$$
s N^{-1} \geqslant \ln N+1 . \quad \mathrm{E} \xi_{2}(H) \rightarrow \lambda, \quad 0<\lambda<\infty .
$$

Toгda

$$
\mathbf{P}\left\{\xi_{2}(H)=m\right\} \rightarrow \frac{\lambda^{m}}{m !} e^{-\lambda}, \quad m=0,1, \ldots
$$

Много сил было приложено к ослаблению условия $s N^{-1} \geqslant \ln N+1$. Это условие приводит к тому, что $s$-цепочка случайно и равновероятно выбранных знаков $N$-буквенного алфавита с гарантированной вероятностью содержит все буквы алфавита. Данное свойство значительно упрощает рассуждения. Лишь совсем недавно В. Г. Михайлову удалось заменить его в теореме для $\xi_{2}\left(S_{N}\right)$ более слабым условием $s N^{-1}-(1 / 4) \ln N \rightarrow \infty$. Статья с доказательством этого результата готовится к печати.

Специальное исследование было посвящено применению при доказательстве предельной теоремы Пуассона для случайных величин $\xi_{r}(H)$ различных вариантов метода Чена-Стейна (см. [46], а также [26, 27]). В работе А. М. Шойтова [40] был рассмотрен случай произвольного натурального $r \geqslant 2$ и были построены оценки для расстояния по вариации между распределением случайной величины $\xi_{r}(H)$ и специально подобранным распределением Пуассона.

Расстояние по вариации между распределениями $F$ и $G$ обозначим через $\rho(F, G)$. Для распределений, сосредоточенных на $\{0,1, \ldots\}$, оно выражается формулой

$$
\rho(F, G)=\frac{1}{2} \sum_{k=0}^{\infty}|F\{k\}-G\{k\}| .
$$


Пусть

$$
(A)_{s}=\left\{\left(a_{1}, \ldots, a_{s}\right) \in A^{s}: a_{i} \neq a_{j}, \text { если }(i \neq j)\right\} .
$$

Обозначим через $\mathrm{M}_{s}(H)$ множество классов $H$-эквивалентности на множестве $A^{s}$. Положим

$$
\begin{array}{ccrl}
\lambda_{r}=\left(\begin{array}{l}
n \\
r
\end{array}\right) \frac{1}{N^{r s}} \sum_{m=1}^{N} \sigma(s, m) & \sum_{x \in \mathrm{M}_{m}(H)}|\varkappa|^{s}, \\
(H)=\frac{|H|}{N^{s}}, & \lambda(r)=\left(\begin{array}{l}
n \\
r
\end{array}\right) \Delta(H)^{r-1}, \\
\psi=(2 s)^{r-1} n \Delta(H) e^{n \Delta(H) / s}, & \Psi=(4 s)^{2 r-1} n \Delta(H) e^{n \Delta(H)} .
\end{array}
$$

Теорема 10. Пусть случайные величины $X_{1}, X_{2}, \ldots, X_{n+s-1}$ распределены на множестве $\{1, \ldots, N\}$ равновероятно. Если выполнено условие

$$
s N^{-1} \geqslant \ln N+1 \text {, }
$$

то $\lambda_{r}<\lambda(r)$, а расстояние по вариачии между распределением случайной величины $\xi_{r}(H)$ и распределением Пуассона с параметром $\lambda_{r}$ не превосходит

$$
\begin{aligned}
\left(\Psi+\lambda_{r}\left(3 \psi+(2 s)^{r}(n \Delta(H))^{r-1}\right)\right. & \left.+12 \lambda(r) N^{-1}\right) \\
& \times\left(\lambda_{r}^{-1}\left(1-e^{-\lambda_{1}}\right)+\psi \min \left\{1,\left(\min \left\{\lambda_{r},\left|\lambda_{r}-\psi\right|\right\}\right)^{-2}\right\}\right) .
\end{aligned}
$$

Из этого утверждения вытекает следующая теорема.

Теорема 11. Пусть случайные величины $X_{t}, t=1, \ldots, n+s-1$, распределены на $\{1, \ldots, N\}$ равновероятно и параметры $n, s \rightarrow \infty$ так, что

$$
s N^{-1}-\ln N \rightarrow \infty, \quad(4 s)^{2 r-1} n \Delta(H) \rightarrow 0
$$

и существует конечный или бесконечный предел $\lambda=\lim \lambda_{r}$.

Если $\lambda<\infty$, то распределение случайной величины $\xi_{r}(H)$ сходится к закону Пуассона с параметром $\lambda$.

Если $\lambda(r) N^{-1} \rightarrow 0 u \lambda=\infty$, то распределение случайной величины $\left(\xi_{r}(H)-\lambda_{r}\right) \lambda_{r}^{-1 / 2}$ сходится к стандартному нормальному закону.

Первая попытка перенесения теоремы 8 на случай неравновероятной последовательности $X_{t}$ была предпринята в [9]. Теорема 3 этой работы описывает достаточные условия выполнения предельной теоремы Пуассона для $\xi_{2}\left(S_{N}\right)$ в ситуации, когда

$$
\left(N \max _{k} \mathbf{P}\left\{X_{t}=k\right\}\right)^{s} \rightarrow 1 \text {. }
$$

Для полиномиальных последовательностей, существенно отличных от равновероятной, условия асимптотической пуассоновости случайной величины $\xi_{2}\left(S_{N}\right)$ указаны в приводимой ниже теореме 13. Они естественным образом переносятся на величины $\xi_{2}(H)$ с произвольным множеством $H$. В этом случае множитель $N$ ! в условии (17) теоремы 13 заменяется на $|H|$.

Исследования в этом направлении велись также в рамках задачи о $H$-связанных цепочках в паре последовательностей (см. раздел 5). 
Укажем одно любопытное свойство, которым обладают случайные величины $\xi_{r}(H)$ при многих больших множествах $H$. Для таких множеств наблюдается сильная взаимная зависимость (вплоть до совпадения) случайных величин $\xi_{r}\left(H_{1}\right)$ и $\xi_{r}\left(H_{2}\right)$ даже при непересекающихся множествах $H_{1}$ и $H_{2}$.

Нам понадобится понятие $m$-транзитивного множества подстановок, которое вполне аналогично понятию $m$-транзитивной группы. Множество $H$ назовем $m$-транзитивным, если для любых двух наборов $x_{1}, \ldots, x_{m}$ и $y_{1}, \ldots, y_{m}$ из попарно различных элементов множества $A$ найдется подстановка $h \in H$, для которой $y_{i}=h\left(x_{i}\right), i=1, \ldots, m$.

Из этого определения следует, что для любого $(N-r)$-транзитивного множества $H$ все $S_{N}$-эквивалентные цепочки, содержащие не более $N-r$ различных букв, $H$-связаны.

Последнее свойство приводит к следующему интересному явлению в случайных последовательностях знаков алфавита $A$.

Пусть, как и раньше, $\xi_{2}(H)$ обозначает число пар $H$-связанных цепочек в последовательности знаков $X_{1}, \ldots, X_{n+s-1}$, выбранных случайно и независимо из алфавита $A$. При определенных условиях для подавляющего большинства пар $S_{N}$-эквивалентных цепочек в цепочках присутствуют не все буквы алфавита. Поэтому числа $\xi_{2}(H)$ для любых $(N-r)$-транзитивных множеств $H$ с малыми значениями параметра $r$ приблизительно одинаковы и близки к $\xi_{2}\left(S_{N}\right)$. А именно, при определенным способом согласованном изменении параметров $n$ и $s$, объема $N$ алфавита, распределения случайных величин $X_{t}$ и изменении самого множества $H$ имеет место соотношение

$$
\mathbf{P}\left\{\xi_{2}(H)=\xi_{2}\left(S_{N}\right)\right\} \rightarrow 1
$$

При этом множество $H$ может существенно отличаться от множества всех подстановок $S_{N}$. Например, при определенных условиях

$$
\mathbf{P}\left\{\xi_{2}\left(A_{N}\right)=\xi_{2}\left(S_{N} \backslash A_{N}\right)=\xi_{2}\left(S_{N}\right)\right\} \rightarrow 1
$$

( $A_{N}$ и $S_{N} \backslash A_{N}$ - множества четных и нечетных подстановок соответственно).

Если же в подавляющем большинстве $S_{N}$-эквивалентных пар цепочки содержат все буквы алфавита, то для любого подмножества $H \subset S_{N}$ (опять речь идет о согласованном изменении параметров, распределения и множества $H$ )

$$
\mathbf{P}\left\{\xi_{2}(H)+\xi_{2}\left(S_{N} \backslash H\right)=\xi_{2}\left(S_{N}\right)\right\} \rightarrow 1
$$

В частности,

$$
\mathbf{P}\left\{\xi_{2}\left(A_{N}\right)+\xi_{2}\left(S_{N} \backslash A_{N}\right)=\xi_{2}\left(S_{N}\right)\right\} \rightarrow 1
$$

Несомненный интерес представляет поведение совместного распределения случайных величин $\xi_{2}\left(S_{N}\right), \xi_{2}(H)$ и $\xi_{2}\left(S_{N} \backslash H\right)$ в промежуточной ситуации, когда пары $S_{N}$-эквивалентных цепочек, содержащих как все, так и и не все буквы алфавита каждая, встречаются в последовательности $X_{t}$ в существенном количестве.

Определим величины

$$
\begin{gathered}
\lambda_{r}(H)=\left(\begin{array}{c}
n-2 s+1 \\
2
\end{array}\right) \mathbf{P}\left\{E_{1,2 s+1}(H) \cap\left\{\left\{\left\{X_{1}, \ldots, X_{s}\right\} \mid=N-r\right\}\right\},\right. \\
\Lambda_{-1}(H)=0, \quad \Lambda_{r}(H)=\lambda_{0}(H)+\ldots+\lambda_{r}(H), \quad r=0,1, \ldots, N-1 .
\end{gathered}
$$


Теорема 12. Пусть $n \rightarrow \infty, s \leqslant n / 2$, параметры $N, s, p_{1}, \ldots, p_{N}$ имножество $H \subseteq S_{N}$ меняются так, что

$$
s|H| P \rightarrow 0
$$

Если при этом

$$
\Lambda_{N-1}(H)-\Lambda_{1}(H) \rightarrow 0,
$$

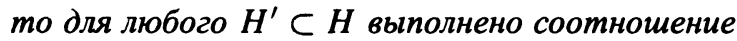

$$
\mathbf{P}\left\{\xi_{2}\left(H^{\prime}\right)+\xi_{2}\left(H \backslash H^{\prime}\right)=\xi_{2}(H)\right\} \rightarrow 1 .
$$

Если же $H^{\prime} \subseteq H \subseteq S_{N}$ является $(N-r)$-транзитивным для некоторого фиксированного натурального числа $r u$

$$
\Lambda_{r-1}(H) \rightarrow 0
$$

mo

$$
\mathbf{P}\left\{\xi_{2}\left(H^{\prime}\right)=\xi_{2}(H)\right\} \rightarrow 1 .
$$

Здесь следует заметить, что

$$
\mathbf{P}\left\{\xi_{2}\left(H^{\prime}\right)+\xi_{2}\left(H \backslash H^{\prime}\right) \geqslant \xi_{2}(H)\right\}=1 .
$$

В случае $H=S_{N}, H^{\prime}=A_{N}$ теорема 12 принимает следующий вид.

Следствие 4. Пусть $n \rightarrow \infty, s \leqslant n / 2$, а параметры $N$ u м меняются так, что выполнено условие

$$
s n N ! N^{-s} \rightarrow 0
$$

Если при этом

$$
\Lambda_{N-1}\left(S_{N}\right)-\Lambda_{1}\left(S_{N}\right) \rightarrow 0,
$$

mo

$$
\mathbf{P}\left\{\xi_{2}\left(A_{N}\right)+\xi_{2}\left(S_{N} \backslash A_{N}\right)=\xi_{2}\left(S_{N}\right)\right\} \rightarrow 1
$$

Если же

$$
\Lambda_{1}\left(S_{N}\right) \rightarrow 0,
$$

mo

$$
\mathbf{P}\left\{\xi_{2}\left(A_{N}\right)=\xi_{2}\left(S_{N} \backslash A_{N}\right)=\xi_{2}\left(S_{N}\right)\right\} \rightarrow 1 .
$$

Это утверждение можно дополнить теоремой, описывающей поведение суммы $\xi_{2}\left(A_{N}\right)+\xi_{2}\left(S_{N} \backslash A_{N}\right)$. Положим

$$
\begin{aligned}
D_{0} & =\mathbf{P}\left\{E_{1,2 s+1}(H)\right\}, \\
D_{k} & =\mathbf{P}\left\{E_{1,2 s+1}(H) E_{1+k .2 s+k+1}(H)\right\}, \quad k=1, \ldots, s-1, \\
D & =D_{1}+\ldots+D_{s-1} .
\end{aligned}
$$

Заметим, что

$$
\Lambda_{N-1}(H)=\left(\begin{array}{c}
n-2 s+1 \\
2
\end{array}\right) D_{0}
$$


Теорема 13. Пусть $n \rightarrow \infty, s \leqslant n / 2, H=S_{N}$, параметры $N, s, p_{1}, \ldots, p_{N}$ меняются так, что выполнено условие

$$
n^{2}\left(D+s N ! P D_{0}\right) \rightarrow 0
$$

Тогда распределение случайного вектора $\left(\xi_{2}\left(S_{N}\right), \xi_{2}\left(A_{N}\right)+\xi_{2}\left(S_{N} \backslash A_{N}\right)\right)$ сближается (в смысле сходимости к нулю расстояния по вариачии) с распределением случайного вектора $\left(\pi_{1}+\pi_{2}, \pi_{1}+2 \pi_{2}\right)$, где $\pi_{1} и \pi_{2}-$ независимые случайные величины, имеючие распределение Пуассона с параметрами $\Lambda_{1}\left(S_{N}\right)$ и $\Lambda_{N-1}\left(S_{N}\right)-\Lambda_{1}\left(S_{N}\right)$ соответственно.

Для равновероятной последовательности можно указать явные выражения для параметров предельного распределения в этом утверждении.

Теорема 14. Пусть случайные величины $X_{t}, t=1, \ldots, n+s-1$, распределены на алфавите А равновероятно: $p_{k}=1 / N, k \in A$, а параметры $N, s, n$ меняются так, что $n \rightarrow \infty, s \leqslant n / 2$ и выполнены условия

$$
\frac{n^{2} N !}{2 N^{s}} \rightarrow \lambda, \quad 0<\lambda<\infty, \quad N e^{-s / N} \rightarrow \gamma, \quad 0<\gamma<\infty
$$

Тогда выполнено заключение теоремы 13, причем

$$
\Lambda_{N-1}\left(S_{N}\right) \rightarrow \lambda \psi(\gamma) e^{-\gamma}, \quad \Lambda_{1}\left(S_{N}\right) \rightarrow \lambda(1+\gamma) e^{-\gamma}
$$

$2 \partial e$

$$
\psi(z)=\sum_{k=0}^{\infty} \frac{z^{k}}{(k !)^{2}}
$$

\section{4. Схема размещения на классах эквивалентности}

В этом разделе рассматривается класс разделимых статистик, определенных на классах $H$-эквивалентных цепочек. В этот класс входят, например, величины $\xi_{r}(H)$ и $\mu_{r}(H)$. Ниже приведены формулы, выражающие разделимые статистики через статистики $\xi_{r}(H)$ и родственные им величины, а также результаты о предельном поведении величин $\xi_{r}(H)$ и $\mu_{r}(H)$, полученные как следствия аналогичных свойств схем конечно-зависимого размещения частиц по ячейкам.

Пусть $X_{1}, X_{2}, \ldots$ - последовательность независимых одинаково распределенных на $\{1, \ldots, N\}$ случайных величин. Пусть $H$ - некоторая группа подстановок на множестве $\{1, \ldots, N\}$.

Отношение $H$-эквивалентности индуцирует разбиение множества $\{1, \ldots, N\}^{s}$ на классы эквивалентных цепочек

$$
\{1, \ldots, N\}^{s}=K_{1} \cup \ldots \cup K_{\left|\mathrm{M}_{s}(H)\right|},
$$

где $\left|\mathrm{M}_{s}(H)\right|-$ число классов эквивалентности.

Пусть

$$
v_{i}(H)=\sum_{j=1}^{n} I\left\{\left(X_{j}, \ldots, X_{j+s-1}\right) \in K_{i}\right\}, \quad i=1, \ldots,\left|\mathrm{M}_{s}(H)\right|
$$


Величина $\nu_{i}(H)$ - это число цепочек в последовательности $X_{1}, \ldots, X_{n+s-1}$, принадлежащих классу $K_{i}$

Будем считать, что $j$-я $s$-цепочка $\left(X_{j}, \ldots, X_{j+s-1}\right)$ помещается в ячейку $K_{i}$, если $\left(X_{j}, \ldots, X_{j+s-1}\right) \in K_{i}$. Определим разделимую статистику формулой

$$
L=\sum_{i=1}^{\left|\mathrm{M}_{s}(H)\right|} f_{\mathbf{i}}\left(v_{i}(H)\right)
$$

где $f_{1}(v), \ldots, f_{\left|\mathrm{M}_{s}(H)\right|}(\nu)$ - набор функщй целочисленного аргумента, удовлетворяющих условию $f_{i}(0)=0, i=1, \ldots,\left|\mathrm{M}_{s}(H)\right|$. Определение (20) аналогично традищионному определению разделимой статистики на частотах цепочек (см., например, [25]) и совпадает с ним при $H=\{e\}$.

Напомним, что понятие разделимой статистики было введено Ю. И. Медведевым в [20]. О свойствах разделимых статистик в равновероятной и полиномиальной схемах размещения см. [3].

В [25] был предложен эффективный метод исследования разделимых статистик на частотах цепочек. Результаты, полученные в [25] с помощью этого метода, были успешно использованы для описания условий асимптотической нормальности статистик $\mu_{r}(\{e\})$ (см. [33, 35, 59]). Некоторые фрагменты рассуждений работы [25] очевидным образом распространяются на наш случай.

По аналогии с событиями $E_{i, j}(H)$ введем события

$$
E_{i_{1}, \ldots, i_{r}}(H)=\left\{\exists h_{2}, \ldots, h_{r} \in H: h_{1+k}\left(x_{i_{1}+j}\right)=x_{i_{k}+j}, j=0,1, \ldots, s-1\right\} .
$$

Очевидно, что

$$
E_{i_{1} \ldots, i_{r}}(H)=E_{i_{1}, i_{2}}(H) \ldots E_{i_{r-1}, i_{r}}(H)=\bigcap_{1 \leqslant k<l \leqslant r} E_{i_{k}, i_{l}}(H) .
$$

Кроме этого, положим

$$
E_{i_{1}, \ldots, i_{r}}(j, H)=E_{i_{1}, \ldots, i_{r}}(H)\left\{\left(X_{i_{1}}, \ldots, X_{i_{1}+s-1}\right) \in K_{j}\right\}
$$

Используя принщип включения-исключения, можно доказать следующее утверждение.

Теорема 15. Разделимая статистика $L$, определенная формулой (20), представляется в виде

$$
L=\sum_{r=1}^{n} \zeta_{r}(H)
$$

где

$$
\begin{aligned}
& \zeta_{r}(H)=\sum_{1 \leqslant i_{1}<\ldots<i_{r} \leqslant n} \sum_{j=1}^{\left|\mathrm{M}_{s}(H)\right|} C_{j}(r) I\left\{E_{i_{1}, \ldots, i_{r}}(j, H)\right\}, \\
& C_{j}(r)=\sum_{l=1}^{r}(-1)^{r-l}\left(\begin{array}{l}
r \\
l
\end{array}\right) f_{j}(l) .
\end{aligned}
$$


Из теоремы 15 следует, что при условии

$$
f_{1}(k)=\ldots=f_{\left|\mathrm{M}_{s}(H)\right|}(k)=f(k), \quad k=0,1, \ldots
$$

(в этом случае статистику $L$ принято называть симметрической разделимой статистикой)

$$
L=\sum_{r=1}^{n}\left(\sum_{l=1}^{r}(-1)^{r-l}\left(\begin{array}{l}
r \\
l
\end{array}\right) f(l)\right) \xi_{r}(H) .
$$

В частности, разделимой статистикой является число $\mu_{r}(H)$ классов эквивалентных цепочек, чьи представители встретились в последовательности $X_{1}, \ldots, X_{n+s-1}$ ровно по $r$ раз для каждого класса. Оно выражается формулой

$$
\mu_{r}(H)=\sum_{k=r}^{n}(-1)^{k-r}\left(\begin{array}{l}
k \\
l
\end{array}\right) \xi_{k}(H) .
$$

Воспользуемся тождеством (24) и результатами раздела 3 о предельном поведении случайных величин $\xi_{r}(H)$ для описания свойств случайных величин $\mu_{r}(H)$. Как и раньше, обозначим через $\mathrm{M}_{s}(H)$ множество классов $H$-эквивалентности на множестве $A^{s}$ и положим

$$
\begin{aligned}
\lambda_{r} & =\left(\begin{array}{l}
n \\
r
\end{array}\right) \frac{1}{N^{r s}} \sum_{m=1}^{N} \sigma(s, m) \sum_{x \in \mathrm{M}_{s}(H)}|x|^{s}, \\
\Delta(H) & =\frac{|H|}{N^{s}} .
\end{aligned}
$$

Из теоремы 11 и тождества (24) вытекает следующее утверждение.

Теорема 16. Пусть случайные величины $X_{t}, t=1, \ldots, n+s-1$, распределены на $\{1, \ldots, N\}$ равновероятно и параметры $n, s$ стремятся к бесконечности так, что

$$
s N^{-1}-\ln N \rightarrow \infty, \quad(4 s)^{2 r+1} n \Delta(H) \rightarrow 0,
$$

и существует конечный предел $\lambda=\lim \lambda_{1}$.

Тогда распределение случайной величины $\mu_{r}(H)$ сходится к закону Пуассона с параметром $\lambda$.

При изучении случайных величин $\xi_{r}(H)$ и $\mu_{r}(H)$ для относительно малых множеств $H$ можно воспользоваться результатами работы [24]. В ней изучались асимптотические свойства статистик $\xi_{r}$ и $\mu_{r}$ в конечно-зависимых схемах случайного размещения частищ по ячейкам. На этом пути В. Г. Михайловым были получены следующие результаты.

Рассмотрим класс групп подстановок, определяемый неравенством

$$
\left|\left\{h \in H: h\left(a_{i}\right)=b_{i}, i=1, \ldots, d\right\}\right| \leqslant 1
$$

для любых наборов $\left(a_{1}, \ldots, a_{d}\right),\left(b_{1}, \ldots, b_{d}\right)$ попарно различных элементов множества $A$. Здесь $d, 1 \leqslant d<s,-$ параметр класса групп. При $d=1$ получаем группы обладающие свойством

$$
|\{h \in H: h(a)=b\}| \leqslant 1 \quad \forall a, b \in A .
$$


Нетрудно заметить, что это будут группы типа латинского прямоугольника.

Число подстановок в любой группе со свойством (25) удовлетворяет условию $|H| \leqslant|A|^{d}$, и при больших значениях $|A|$ в нее входит лишь незначительная часть из общего числа $|A|$ ! подстановок.

Нам понадобится вспомогательное отношение эквивалентности, которое мы введем следующим образом. Разобьем множество $A^{s}$ всех $s$-цепочек на множества

$$
A_{0}^{s}=\left\{\left(a_{1}, \ldots, a_{s}\right) \in A^{s}: \text { все элементы } a_{1}, \ldots, a_{d} \text { различны }\right\}
$$

и $A_{1}^{s}=A^{s} \backslash A_{0}^{s}$. Любой класс $H$-эквивалентности целиком лежит либо в $A_{0}^{s}$, либо в $A_{1}^{s}$. Поэтому можно построить следующее разбиение множества $A^{s}$ на классы эквивалентности. На множестве $A_{0}^{s}$ в качестве классов эквивалентности отношения $\phi(d, H)$ возьмем классы отношения $H$-эквивалентности. На множестве $A_{1}^{s}$ в качестве классов эквивалентности отношения $\phi(d, H)$ возьмем сами цепочки. Цепочки, принадлежащие одному классу $\phi(d, H)$-эквивалентности, будем называть $(d, H)$-эквивалентными.

Пусть $X_{1}, X_{2}, \ldots$ - последовательность независимых случайных величин, принимающих значения из конечного множества $A$,

$$
\mathbf{P}\left\{X_{i}=a\right\}=p_{i, a}, \quad a \in A, \quad p=\max _{i \geqslant 1, a \in A} p_{i, a} .
$$

Пусть $H$ - группа подстановок на множестве $A$. Обозначим через $\xi_{r}(H)$ число $r$-наборов $H$-эквивалентных $s$-цепочек в последовательности $X_{1}, X_{2}, \ldots, X_{n+s-1}$. Через $\mu_{r}(H)$ обозначим число классов $\phi(H)$-эквивалентности, представители которых встретились среди $s$-цепочек последовательности $X_{1}, X_{2}, \ldots, X_{n+s-1}$ ровно по $r$ раз.

Аналогичным образом для отношения $\Phi(d, H)$-эквивалентности определим величины $\xi_{r}(d, H)$ и $\mu_{r}(d, H)$.

Теорема 17. Пусть $r \geqslant 2$ фиксировано, а параметры $n, s, d$, алфавит $A$, группа $H u$ распределения случайных величин $X_{1}, \ldots, X_{n+s-1}$ меняются так, что группа Н удовлетворяет соотночению (25) $u$

$$
\begin{gathered}
\mathrm{E} \xi_{r}(d, H) \rightarrow \infty, \quad \ln s=o\left(\ln \mathrm{E} \xi_{r}(d, H)\right), \\
s p+s|H| p^{s-d+1}+s|H| n p^{s} \rightarrow 0 .
\end{gathered}
$$

Тогда функция распределения случайной величины $\left(\xi_{r}(d, H)-\mathbf{E} \xi_{r}(d, H)\right)\left(\mathbf{D} \xi_{r}(d, H)\right)^{-1 / 2}$ сходится $\kappa \Phi(x)$.

Теорема 18. Пусть $r \geqslant 2$ фиксировано, а параметры $n, s, d$, алфавит $A$, группа $H u$ распределения случайных величин $X_{1}, \ldots, X_{n+s-1}$ меняются так, что группа $H$ удовлетворяет соотношению (25) $u$

$$
\begin{gathered}
\mathrm{E} \mu_{r}(d, H) \rightarrow \infty, \quad \ln s=o\left(\ln \mathrm{E} \mu_{r}(d, H)\right), \\
s^{r+1}\left(p+|H| p^{s-d+1}+|H| n p^{s}\right) \rightarrow 0 .
\end{gathered}
$$

Тогда функция распределения случайной величины $\left(\mu_{r}(d, H)-\mathbf{E} \mu_{r}(d, H)\right)\left(\mathbf{D} \mu_{r}(d, H)\right)^{-1 / 2}$ сходится $\kappa \Phi(x)$.

Нетрудно заметить, что при $d=1$ (то есть для групп типа латинского прямоугольника) отношения $\phi(d, H)$-эквивалентности и $\phi(H)$-эквивалентности совпадают. Поэтому из теорем 17 и 18 вытекают следующие утверждения. 
Следствие 5. Пусть $r \geqslant 2$ фиксировано, параметры $n, s$, алфавит A, группа $H$ удовлетворяет соотночению (26) и вместе с распределением случайных величин $X_{1}, \ldots, X_{n+s-1}$ меняется так, что

$$
\mathrm{E} \xi_{r}(H) \rightarrow \infty, \quad \ln s=o\left(\ln \mathrm{E} \xi_{r}(H)\right), \quad s\left(p+|H| n p^{s}\right) \rightarrow 0 .
$$

Тогда функиия распределения случайной величины $\left(\xi_{r}(H)-\mathbf{E} \xi_{r}(H)\right)\left(\mathbf{D} \xi_{r}(H)\right)^{-1 / 2}$ сходится $\kappa \Phi(x)$.

Следствие 6. Пусть $r \geqslant 2$ фиксировано, параметры $n, s$, алфавит $A$, группа Н удовлетворяет соотношению (26) и вместе с распределением случайных величин $X_{1}, \ldots, X_{n+s-1}$ меняется так, что

$$
\mathbf{E} \mu_{r}(H) \rightarrow \infty, \quad \ln s=o\left(\ln \mathbf{E} \mu_{r}(H)\right), \quad s^{r+1}\left(p+|H| n p^{s}\right) \rightarrow 0
$$

Тогда функиия распределения случайной величины $\left(\mu_{r}(H)-\mathbf{E} \mu_{r}(H)\right)\left(\mathbf{D} \mu_{r}(H)\right)^{-1 / 2}$ cходится $\kappa \Phi(x)$.

Замечание 4. Пусть группа $H$ является латинским прямоугольником, случайные величины $X_{1}, \ldots, X_{n+s-1}$ распределены на множестве $A$ равномерно: $p_{a}=1 /|A|, a=1, \ldots,|A|$, и выполнены условия (27). Тогда

$$
\begin{aligned}
& \mathbf{E} \xi_{r}(H)=\left(\begin{array}{l}
n \\
r
\end{array}\right)\left(|H||A|^{-s}\right)^{r-1}(1+o(1)), \\
& \mathbf{E} \mu_{r}(H)=\left(\begin{array}{l}
n \\
r
\end{array}\right)\left(|H \| A|^{-s}\right)^{r-1}(1+o(1)) .
\end{aligned}
$$

Если при этом $s$ фиксировано, то условия следствий 5 и 6 принимают вид

$$
n,|A| \rightarrow \infty, \quad\left(\begin{array}{l}
n \\
r
\end{array}\right)\left(|H \| A|^{-s}\right)^{r-1} \rightarrow \infty, \quad n|H \| A|^{-s} \rightarrow 0 .
$$

Перейдем к изучению величин $\xi_{r}(H)$ в случае, когда $d \geqslant 2$. Будем считать, что параметры $s, d$ при переходе к пределу остаются неизменными, а вероятности исходов пропорциональны друг другу:

$$
0<c_{1} \leqslant|A| p_{i . a} \leqslant c_{2}<\infty, \quad i=1,2 \ldots, \quad a \in A .
$$

где $c_{1}, c_{2}$ - некоторые постоянные. Такую полиномиальную схему будем называть меняющейся регулярно или просто регулярной полиномиальной схемой.

Теорема 19. Пусть параметры $r \geqslant 2, s, d \geqslant 2$ остаются неизменными, параметр $n$, алфавит А и распределения случайных величин $X_{1}, \ldots, X_{n+s-1}$ меняются так, что выполнено условие регулярности (28), группа $H$, изменяясь, удовлетворяет соотношению (25) $u$

$$
\begin{gathered}
\mathbf{E} \xi_{r}(H) \rightarrow \infty, \quad|A|^{-2} \mathbf{E} \xi_{r}(H) \rightarrow 0, \\
|H \| A|^{-s}\left(n+|A|^{d-1}\right) \rightarrow 0 .
\end{gathered}
$$

Тогда функиия распределения случайной величины $\left(\xi_{r}(H)-\mathbf{E} \xi_{r}(H)\right)\left(\mathbf{D} \xi_{r}(d, H)\right)^{-1 / 2}$ cходится $\kappa \Phi(x)$.

Свойства зависимых схем размещения изучались в $[13,14]$. Идеи и результаты этих работ также могут оказаться полезными при изучении свойств $H$-эквивалентных цепочек. 


\section{5. Связанные цепочки из разных последовательностей}

В отличие от рассмотренных выше задач, в этом разделе нас будут интересовать пары эквивалентных и $\boldsymbol{H}$-эквивалентных цепочек в случае, когда одна цепочка расположена в одной последовательности, а вторая - в другой. Эта постановка хороша тем, что в ней естественно рассматривать и случай, когда распределение величин одной последовательности отличается от распределения величин в другой, а это существенно расширяет круг содержательных задач.

Итак, пусть $X=\left(X_{1}, \ldots, X_{m}\right)$ и $Y=\left(Y_{1}, \ldots, Y_{n}\right)$ - две последовательности независимых одинаково (внутри каждой последовательности) распределенных случайных величин, принимающих значения в множестве $\{1,2, \ldots\}$,

$$
\begin{aligned}
& p_{k}=\mathbf{P}\left\{X_{1}=k\right\}, \quad q_{k}=\mathbf{P}\left\{Y_{1}=k\right\}, \quad k=1,2, \ldots, \\
& p=\max _{k} p_{k}, \quad q=\max _{k} q_{k}, \quad P=m p^{s}, \quad Q=n q^{s} .
\end{aligned}
$$

Пусть задан конечный набор $H$ взаимно однозначных отображений $h:\{1,2, \ldots\} \rightarrow$ $\{1,2, \ldots\}$. Введем обозначения

$$
\begin{aligned}
E_{i, j}(h) & =\left\{\left(h\left(X_{i}\right), \ldots, h\left(X_{i+s-1}\right)\right)=\left(Y_{j}, \ldots, Y_{j+s-1}\right)\right\} \\
E_{i, j}(H) & =\bigcup_{h \in H} E_{i, j}(h) .
\end{aligned}
$$

Число пар $H$-связанных цепочек из последовательностей $X$ и $Y$ выражается формулой

$$
N(H)=\sum_{i=1}^{m} \sum_{j=1}^{n} I\left(E_{i, j}(H)\right)
$$

(здесь и далее выражение $I(\cdot)$ обозначает индикатор случайного события или неслучайного множества). В этом определении считается, что

$$
X_{m+i}=X_{i}, \quad Y_{n+i}=Y_{i}, \quad i=1,2, \ldots
$$

Это предположение позволяет отчасти упростить выкладки, но не влияет на вид предельного распределения.

Введем обозначение

$$
D_{k}=\mathbf{P}\left\{E_{1,1}(H) E_{1+k, 1+k}(H)\right\}, \quad k=0,1, \ldots, s-1 .
$$

В частности,

$$
\left.\mathbf{P}\left\{E_{1,1}(H)\right\}=D_{0}=\mathbf{P}\left\{E_{1,1}(H) E_{1,1}(H)\right\}\right\} .
$$

Пусть

$$
D=D_{1}+\ldots+D_{s-1}
$$

Распределение Пуассона с параметром $\lambda$ будем обозначать Ро $(\lambda)$. Через $\mathscr{L}(W)$ будем обозначать распределение случайной величины $W$.

Приводимая далее теорема получена в [31]. 
Теорема 20. Пусть параметры схемы $m, n \rightarrow \infty, a p_{k}, q_{k}, k=1,2, \ldots$, меняются так, чmo

$$
\begin{gathered}
\mathbf{E} N(H)=m n D_{0} \rightarrow \lambda<\infty, \\
m n D+s|H|(P+Q) \rightarrow 0 .
\end{gathered}
$$

Тогда

$$
\mathscr{L}(N(H)) \rightarrow \operatorname{Po}(\lambda)
$$

Замечание 5. Доказательство теоремы 20 проводится с помощью локального варианта метода Чена-Стейна. Этот метод позволяет получить явную оценку расстояния по вариации между распределением величины $N(H)$ и пуассоновским распределением с параметром $\mathrm{E} N(H)$ (см. теорему 22 ниже). Из этой оценки кроме условий асимптотической пуассоновости можно также получить достаточные условия асимптотический нормальности величины $N(H)$ (см. теорему 24 ниже).

Замечание 6. Многими авторами (см. [29, 43-45, 47-57]) исследовалась случайная величина $N(\{e\})$ - число точных совпадений $s$-цепочек - и некоторые связанные с ней случайные величины. При $H=\{e\}$ условие (30) (с учетом других условий теоремы) принимает вид $s(P+Q) \rightarrow 0$.

Теорема 20 позволяет исследовать число пар $H$-эквивалентных цепочек как при малых, так и больших множествах $H$.

Пусть распределения $\left\{p_{k}\right\}$ и $\left\{q_{k}\right\}$ сосредоточены на множестве $\{1, \ldots, N\}$, причем распределение $\left\{q_{k}\right\}$ на нем равновероятно: $q_{1}=\ldots=q_{N}=1 / N$. Тогда

$$
D_{k} \leqslant \frac{|H|}{N^{s+k}}, \quad k=0,1, \ldots, s-1, \quad D<\frac{|H|}{N^{s}(N-1)} .
$$

Если при этом множество $H$ удовлетворяет условию (26) (то есть $H$ - множество типа латинского прямоугольника), то

$$
D_{k}=|H| N^{-s-k}, \quad k=0,1, \ldots, s-1 .
$$

Используя эти свойства, из теоремы 20 нетрудно получить следующее утверждение.

Теорема 21. Пусть элементы последовательностей $X$ и $Y$ распределены на множестве $\{1, \ldots, N\}$ равновероятно: $p_{k}=q_{k}=1 / N, k=1, \ldots, N$, а множество $Н$ удовлетворяет условию (26). Пусть $n \rightarrow \infty$, а остальные параметры схемы меняются так, что

$$
\frac{\ln m}{n}+\frac{\ln n}{m}=O(1)
$$

и выполняется условие (29).

Тогда распределение случайной величины $N(H)$ сходится к распределению Пуассона с параметром $\lambda$.

Условие (32) гарантирует определенного рода соразмерность длин последовательностей $X$ и $Y$.

Приведем оценки скорости сходимости, полученные в работе [31]. Обозначим через $\rho(F, G)$ расстояние по вариации между распределениями $F$ и $G$. 
Теорема 22. Пусть $m, n \geqslant 2 s-1$. Тогда

$$
\begin{aligned}
\left.\rho(\mathscr{L}(N(H))), \operatorname{Po}\left(m n D_{0}\right)\right) \leqslant & \left(1-e^{-m n D_{0}}\right)\left(2 m n D\left(m n D_{0}\right)^{-1}\right. \\
& \left.+2(2 s-1)|H|(P+Q)+(2 s-1)^{2}\left(m n D_{0}\right)^{-1}|H|^{2} P Q\right) .
\end{aligned}
$$

Обозначим через $W$ набор случайных величин $\left\{I\left(E_{i, j}(H)\right)\right\}_{\Gamma}$ на множестве

$$
\Gamma=\{1, \ldots, m\} \times\{1, \ldots, n\} .
$$

Пусть $\pi=\left\{\pi_{i, j}\right\}_{\Gamma}-$ набор независимых случайных величин, имеющих распределения Пуассона с параметрами

$$
\mathbf{E} \pi_{i, j}=\mathbf{P}\left\{E_{i, j}(H)\right\} .
$$

Теорема 23. Пусть $m, n \geqslant 2 s-1$. Тогда

$$
\rho(\mathscr{L}(W), \mathscr{L}(\pi)) \leqslant 2 m n D+2(2 s-1) m n D_{0}|H|(P+Q)+(2 s-1)^{2}|H|^{2} P Q .
$$

Из теоремы 23 следуют теорема 20 и следующая теорема 24.

Теорема 24. Пусть параметры схемы $m, n \rightarrow \infty, a p_{k}, q_{k}, k=1, \ldots, N$, меняются так, что

$$
\begin{gathered}
\mathrm{E} N(H)=m n D_{0} \rightarrow \infty, \\
D D_{0}^{-1}+s|H|(P+Q) \rightarrow 0 .
\end{gathered}
$$

Тогда функиия распределения случайной величины $\left(m n D_{0}\right)^{-1 / 2}\left(N(H)-m n D_{0}\right)$ сходится $\kappa$ стандартной нормальной функиии распределения.

Как недавно показал В. Г. Михайлов, в случае, когда равновероятными являются обе последовательности, условия сходимости распределения величины $N\left(S_{N}\right)$ к распределению Пуассона выглядят следующим образом.

Теорема 25. Пусть элементы последовательностей $X$ и $Y$ независимы и распределены на множестве $\{1, \ldots, N\}$ равновероятно. Если $m, n, N, s \rightarrow \infty$ так, что величины $n$ и $m$ имеют один порядок роста и выполнены условие (29) и условие

$$
\frac{s}{N}-\frac{1}{4} \ln N \rightarrow \infty
$$

mo

$$
\mathscr{L}\left(N\left(S_{N}\right)\right) \rightarrow \operatorname{Po}(\lambda) .
$$

Приведем теперь результаты исследования асимптотических свойств вероятностей $\mathbf{P}\left\{E_{i, j}\left(S_{N}\right)\right\}$.

Рассмотрим две последовательности независимых одинаково распределенных внутри каждой последовательности случайных величин $X_{1}, \ldots, X_{s}$ и $Y_{1}, \ldots, Y_{s}$ со значениями в множестве $\{1, \ldots, N\}$. Нас будет интересовать вопрос о величине вероятности события

$$
E(s, N)=\left\{\exists \sigma \in S_{N}: Y_{k}=\sigma\left(X_{k}\right), k=1, \ldots, s\right\},
$$

где $S_{N}$ - множество всех подстановок на $\{1, \ldots, N\}$. 
Теорема 26. Пусть $\mu(s)$ - число различных значений, принятых величинами $X_{1}, \ldots, X_{s}$,

$$
\mathbf{P}\left\{Y_{i}=k\right\}=\frac{1}{N}, \quad k=1, \ldots, N, \quad i=1, \ldots, n .
$$

Tогда

$$
\mathbf{P}\{E(s, N)\}=N^{-s} \mathbf{E}\left((N)_{\mu(s)}\right) .
$$

Соотношение (38) обобщает использованное в [9] равенство

$$
\mathbf{P}\left\{E_{1,1}\left(S_{N}\right)\right\}=N^{-2 s} \sum_{m=1}^{\min \{s, N\}} \sigma(s, m)\left((N)_{m}\right)^{2}
$$

для полностью равновероятного случая.

Связь между равенствами (38) и (39) становится прозрачной, если вспомнить, что в равновероятной полиномиальной схеме с $s$ испытаниями и $N$ исходами

$$
\mathbf{P}\{\mu(s)=m\}=\sigma(s, m)(N)_{m} N^{-s}, \quad m=1, \ldots, s .
$$

Напомним, что числа Стирлинга второго рода $\sigma(s, m)$ определяются как коэффициенты в системе тождеств

$$
x^{k}=\sum_{m=0}^{k} \sigma(k, m)(x)_{m}, \quad k=0,1, \ldots
$$

Замечание 7. Аналогичным равенству (38) образом можно выразить вероятность события

$$
F(s, N)=\left\{X_{i}=X_{j} \Longrightarrow Y_{i}=Y_{j}, 1 \leqslant i<j \leqslant s\right\} .
$$

В этом случае вместо (38) получаем равенство

$$
\mathbf{P}\{F(s, N)\}=N^{-s} \mathbf{E}\left(N^{\mu(s)}\right) .
$$

То же самое можно сказать про вероятности событий более сложного вида. Пусть помимо последовательности $X_{1}, \ldots, X_{n}$ заданы $r+t$ независимых последовательностей $Y_{1}^{u}, \ldots, Y_{n}^{u}, u=1, \ldots, r+t$, независимых случайных величин, распределенных на множестве $\{1, \ldots, N\}$ равновероятно. Пусть

$$
\begin{aligned}
& E^{t}(s, N)=\left\{X_{i}=X_{j} \Longleftrightarrow Y_{i}^{t}=Y_{j}^{t}, 1 \leqslant i<j \leqslant s\right\}, \\
& F^{t}(s, N)=\left\{X_{i}=X_{j} \Longrightarrow Y_{i}^{t}=Y_{j}^{t}, 1 \leqslant i<j \leqslant s\right\} .
\end{aligned}
$$

Тогда для вероятности события

$$
E^{r} F^{t}(s, N)=E^{1}(s, N) \ldots E^{r}(s, N) F^{r+1}(s, N) \ldots F^{r+t}(s, N)
$$

выполнено равенство

$$
\mathbf{P}\left\{E^{r} F^{t}(s, N)\right\}=N^{-(r+t) s} \mathbf{E}\left(\left(N_{\mu(s)}\right)^{r} N^{t \mu(s)}\right) .
$$


Важным следствием теоремы 26 является система неравенств

$$
\mathbf{P}\{\mu(s)=s\} \leqslant \frac{N^{s}}{(N)_{s}} \mathbf{P}\{E(s, N)\} \leqslant \mathbf{P}\{\mu(s)=s\}+\frac{1}{N-s} .
$$

В равновероятном случае из нее получаем систему неравенств

$$
\left(\frac{(N)_{s}}{N^{s}}\right)^{2} \leqslant \mathbf{P}\{E(s, N)\} \leqslant \frac{(N)_{s}}{N^{s}}\left(\frac{(N)_{s}}{N^{s}}+\frac{1}{N-s}\right) .
$$

Поэтому при $s / N \rightarrow 0$

$$
\mathbf{P}\{E(s, N)\}=\left(\frac{(N)_{s}}{N^{s}}\right)^{2}\left(1+O\left(\frac{1}{N-s}\right)\right) .
$$

Благодаря цепочке неравенств (40) удается исследовать асимптотику вероятности $\mathbf{P}\{E(s, N)\}$ и при условии (37). Так, (40), предельная теорема Пуассона и оценки точности пуассоновской аппроксимации для числа пустых ячеек в полиномиальной схеме размещения (см. [1], с. 118-119, и [16]), а также экстремальный характер распределения числа занятых ячеек в равновероятной схеме размещения (см. [18]) дают следующий результат.

Положим

$$
P_{r}=\sum_{k=1}^{N} p_{k}^{r}, \quad p=\max _{k} p_{k}
$$

Теорема 27. Пусть случайные величины $X_{1}, \ldots, X_{s}$ имеют произвольное полиномиальное распределение, а для $Y_{1}, \ldots, Y_{s}$ выполнено условие (37).

тогда, если $s^{2} P_{2} \rightarrow 0$, mo

$$
\mathbf{P}\{E(s, N)\}=1+O\left(s^{2} P_{2}\right)
$$

если $s p\left(1+s^{2} P_{2}\right) \rightarrow 0$, mo

$$
\mathbf{P}\{E(s, N)\}=\exp \left\{-\frac{s^{2}}{2}\left(P_{2}+\frac{1}{N}\right)\right\}\left(1+O\left(s p\left(1+s^{2} P_{2}\right)\right)\right) .
$$

Замечание 8. Для случая, когда знаки цепочек $X$ и $Y$ распределены равновероятно, можно указать простую рекуррентную процедуру вычисления $\mathbf{P}\{E(s, N)\}$. Пусть

$$
z_{1}(1)=1, \quad z_{2}(1)=\ldots=z_{N}(1)=0, \quad z_{1}(s)=z_{1}(s-1) N^{-2}
$$

при $s=2, \ldots, N$ и

$$
z_{k}(s)=z_{k-1}(s-1)\left(1-\frac{k-1}{N}\right)^{2}+z_{k}(s-1) \frac{k}{N^{2}}, \quad k=2, \ldots, N .
$$

Тогда

$$
\mathbf{P}\{E(s, N)\}=z_{1}(N)+\ldots+z_{N}(N) .
$$




\section{6. Перестановочная эквивалентность}

Пусть $s$ - натуральное число и $G$ - подгруппа симметрической группы подстановок $S_{s}$. Два вектора ( $s$-цепочки) $\bar{a}=\left(a_{1}, \ldots, a_{s}\right)$ и $\bar{b}=\left(b_{1}, \ldots, b_{s}\right)$ из $A^{s}$ будем называть перестановочно эквивалентными относительно группы $G$ ( $G$-эквивалентными в соответствии с [2]), если существует такая подстановка $g$ из $G$, что $a_{g(j)}=b_{j}$ для всех $j=1, \ldots, s$. В этом случае будем писать $\bar{a} G \bar{b}$. В частности, запись $\bar{a}\{e\} \bar{b}$, где $e-$ единичная подстановка, означает, что $\bar{a}=\bar{b}$.

Если $G_{1} \subseteq G_{2} \subseteq S_{s}$, то из $\bar{a} G_{1} \bar{b}$ следует, что $\bar{a} G_{2} \bar{b}$ и $\bar{a} S_{s} \bar{b}$. Последнее соотношение эквивалентно условию, что для каждого знака алфавита частоты его появления в цепочках $\bar{a}$ и $\bar{b}$ одинаковы. Поэтому таким же свойством обладают $G$-эквивалентные $s$-цепочки при любой группе $G$.

Рассмотрим следующую задачу. Имеются две последовательности $X_{1}, \ldots, X_{n+s-1}$ и $Y_{1}, \ldots, Y_{n}$ знаков алфавита $A$. Требуется проверить гипотезу о том, что последовательность $Y_{1}, \ldots, Y_{n}$ получена по правилу

$$
Y_{t}=f\left(X_{t}, \ldots, X_{t+s-1}\right), \quad t=1,2, \ldots, n,
$$

с некоторой (неизвестной) функцией $f\left(x_{1}, \ldots, x_{s}\right): A^{s} \rightarrow A$. В этом случае применим критерий совпадений, состоящий в проверке свойства

$$
\left\{\left(X_{i}, \ldots, X_{i+s-1}\right)=\left(X_{j}, \ldots, X_{j+s-1}\right)\right\} \Longrightarrow Y_{i}=Y_{j}
$$

для всех пар $1 \leqslant i<j \leqslant n$.

Если заранее известно, что функция $g$ обладает свойством

$$
f\left(x_{1}, \ldots, x_{s}\right)=f\left(x_{g(1)}, \ldots, x_{g(s)}\right)
$$

при всех $x_{1}, \ldots, x_{s} \in A$ и $g \in G$, где $G$ - некоторая группа подстановок на множестве $\{1,2, \ldots, s\}$, то правило (41) можно заметить правилом

$$
\left\{\left(X_{i}, \ldots, X_{i+s-1}\right) G\left(X_{j}, \ldots, X_{j+s-1}\right)\right\} \Longrightarrow Y_{i}=Y_{j}
$$

Выигрыш состоит в том, что событие, указанное в левой части соотношения (42), встречается в случайной последовательности гораздо чаще, чем совпадение двух $s$-цепочек.

Критерии, использующие правила (41) и (42), удобны тем, что они не могут отвергнуть гипотезу наличия функциональной зависимости (во втором случае - функциональной зависимости указанного выше вида), если она верна. Поэтому они характеризуются лишь вероятностью ошибочного принятия гипотезы о зависимости.

Эта вероятность в значительной степени определяется распределением случайной величины

$$
\zeta_{2}(G)=\sum_{1 \leqslant i<j \leqslant n} I\left\{\left(X_{i}, \ldots, X_{i+s-1}\right) G\left(X_{j}, \ldots, X_{j+s-1}\right)\right\},
$$

равной числу пар $G$-эквивалентных $s$-цепочек в последовательности $X$.

Оказалось, что случайная величина $\zeta_{2}(G)$ обладает весьма любопытными асимптотическими свойствами. А. М. Шойтов показал [37], что при определенных условиях значение случайной величины $\zeta_{2}(G)$ совпадает в пределе с числом событий $\left\{X_{i}=X_{i+s}\right\}$. Его результат можно сформулировать следующим образом. 
Обозначим через $T$ циклический сдвиг на один шаг (в любую сторону) на множестве $\{1,2, \ldots, s\}$. Как и раньше, через $\mathscr{L}(W)$ будем обозначать распределение случайной величины $W$. Распределение Пуассона с параметром $\lambda$ будем обозначать $\operatorname{Po}(\lambda)$, а функцию распределения нормального закона $-\Phi(x)$. Пусть еще

$$
P_{r}=\sum_{a \in A} p_{a}^{r}
$$

Теорема 28. Пусть $n \rightarrow \infty$, а параметры $s, N$, вектор вероятностей $\mathscr{P}$ и группа $G$ меняются так, что $T \in G, s \geqslant 3, s P_{2}<e^{c}$ для некоторой постоянной $c<1$.

Тогда если $п P_{2}=O(1)$, то

$$
\mathbf{P}\left\{\zeta_{2}(G)=\sum_{i=1}^{n-1} I\left\{X_{i}=X_{i+s}\right\}\right\} \rightarrow 1
$$

а распределение случайной величины $\zeta_{2}(G)$ сближается с распределением Пуассона $c$ параметром $n P_{2}$ :

$$
d\left(\mathscr{L}\left(\zeta_{2}(G)\right), \operatorname{Po}\left(n P_{2}\right)\right)=O\left(P_{3} P_{2}^{-1}\right)
$$

если $n P_{2} \rightarrow \infty u n^{3} P_{2}^{7} \rightarrow 0$ (при $\left.s \geqslant 4\right), n^{3} P_{2}^{5} \rightarrow 0$ (при $\left.s=3\right)$, то для любого фиксированного $x \in \mathbf{R}$

$$
\mathbf{P}\left\{\frac{\zeta_{2}(G)-n P_{2}}{\sqrt{n P_{2}}} \leqslant x\right\} \rightarrow \Phi(x) .
$$

Замечание 9. При доказательстве теоремы 28 показывается, что решающий вклад в поведение случайной величины $\zeta_{2}(G)$ (см. формулу (43)) вносят слагаемые вида

$$
I\left\{\left(X_{i}, \ldots, X_{i+s-1}\right) G\left(X_{i+1}, \ldots, X_{i+s}\right)\right\} .
$$

Нетрудно проверить, что при условии $T \in G$ эти слагаемые имеют смысл $I\left\{X_{i}=X_{i+s}\right\}$. В итоге оказывается, что в рассмотренном случае главный член асимптотики случайной величины $\zeta_{2}(G)$ не зависит от группы $G$.

Замечание 10. Из суммы (43) можно исключить слагаемые при $j-i \leqslant s-1$, относящиеся к перекрывающимся цепочкам. Тогда основной вклад в асимптотическое поведение величины $\zeta_{2}(G)$ будут вносить члены, зависящие от вида группы $G$. Но и в этом случае, как показывает приводимая ниже теорема 29, доказанная недавно А. М. Шойтовым, наблюдаются характерные особенности той же самой природы в асимптотическом поведении $\zeta_{2}(G)$.

Замечание 11. Теорема 28 описывает практически весь диапазон изменения параметров схемы $X$, кроме вырожденного случая $s \leqslant 2$ и случая $s P_{2}>e$, для которого возможна сходимость распределения случайной величины $\zeta_{2}(G)$ к закону Пуассона. В случае $s=2$ можно доказать, что предельным распределением для $\zeta_{2}(G)$ будет распределение Пуассона с параметром $2 \lambda$.

Рассмотрим случайную величину

$$
\zeta_{2}^{\prime}(G)=\sum_{1 \leqslant i_{1}<i_{1}+n \leqslant i_{2} \leqslant N} I\left(\left(X_{i_{1}}, \ldots, X_{i_{1}+n-1}\right) G\left(X_{i_{2}}, \ldots, X_{i_{2}+n-1}\right)\right),
$$


равную числу пар неперекрывающихся $G$-эквивалентных $s$-цепочек в последовательности $X$. Пусть

$$
\lambda(n)=\left(1-\frac{1}{N}\right)^{2}\left(\begin{array}{l}
n \\
2
\end{array}\right) \frac{s}{N^{s}} .
$$

Обозначим через $T(s)$ группу всех циклических сдвигов на множестве $\{1,2, \ldots, s\}$.

Теорема 29. Пусть случайные величины $X_{1}, \ldots, X_{n+s-1}$ распределены независимо и равномерно на A, а параметры схемы меняются так, что $n, s \rightarrow \infty u \lambda(n) \rightarrow \lambda, 0<\lambda<\infty$. Тогда

(a) если $N \rightarrow \infty$, то

$$
\mathscr{L}\left(\zeta_{2}^{\prime}(T(s))\right) \rightarrow \pi(\lambda)
$$

(б) если параметр $N$ остается неизиенным, то распределение случайной величины $\zeta_{2}^{\prime}(T(s))$ сходится к распределению суммы $u_{1} v_{1}+u_{2} v_{2}+\ldots+u_{\pi} v_{\pi}$, где случайные величины $\pi, u_{k} u v_{k}, k=1,2, \ldots$ независимы, величина $\pi$ распределена по закону Пуассона с параметром $\lambda$, а величины $u_{k} u v_{k}, k=1,2, \ldots$, имеют геометрическое распределение с вероятностью успеха $1-N^{-1}$.

Замечание 12. Причина возникновения в пределе такого зкзотического распределения состоит в следующем. При неизменном числе исходов события вида (44) группируются в отрезки периодических (с периодом длины $s$ ) последовательностей. Число событий вида (44) в таком отрезке имеет в пределе геометрическое распределение. Число отрезков является суммой редких событий и имеет в пределе распределение Пуассона. События из одного отрезка периодичности не вносят вклада в сумму (43), если только отрезок не слишком длинный (вероятность такого события пренебрежимо мала), поскольку они перекрываются. Но пары $T(s)$-эквивалентных цепочек по одной из двух разных отрезков периодичности уже будут подсчитаны. Они дадут вклад, равный произведению числа цепочек в каждом из отрезков. Так возникает предельное распределение в п. (б) теоремы 29.

\section{Список литературы}

1. Колчин В. Ф., Севастьянов Б. А., Чистяков В. П., Случайные размещения. Наука, Москва, 1976.

2. Сачков В. Н., Введение в комбинаторные методы дискретной математики. Наука, Москва, 1982.

3. Иванов В. А., Ивченко Г. И., Медведев Ю. И., Дискретные задачи теории вероятностей. Итоги науки и техники. Сер. Теория вероятностей. Математическая статистика. Теоретическая кибернетика. (1984) 22, 3-60.

4. Буравлев С. М., Пуассоновское предельное распределение числа отрезков, связанных подстановками из симметрической группы, в последовательности независимых равновероятных испытаний. В кн.: Вторая Всероссийская школа-коллоквиум по стохастическим методам. Тезисы докладов. ТВП, Москва, 1995, с. 28-30.

5. Буравлев С. М., Некоторые алгоритмы подсчета статистик, зависящих от числа пар отрезков последовательности, связанных подстановками. Обозрение прикладной и промышленной математики (1997) 4, №3, 331-333.

6. Буравлев С. М., Предельные теоремы для случайных величин от числа пар отрезков, связанных подстановками из латинского прямоугольника. В кн.: Третья Всероссийская школа-коллоквиум по стохастическим методам. Тезисы докладов. ТВП, Москва, 1996, с. 37-40. 
7. Буравлев С. М., Локальная асимптотическая нормальность числа пар отрезков последовательности, связанных подстановками из латинского прямоугольника. Обозрение прикладной и промыниленной математики (1998) 5, №2, 205-207.

8. Буравлев С. М., Уточнение одного предельного распределения. Обозрение прикладной и промышиенной математики (1999) 6, №1, 127-128.

9. Буравлев С. М., Повторения с точностью до перестановок в последовательности независимых испытаний. Дискретная математика (1999) 11, №1, 53-75.

10. Буравлев С. М., Повторения с точностью до перестановок, образующих латинский прямоугольник. Дискретная математика (2000) 12, №1, 24-46.

11. Гусев В. Д., Немытикова Л. А., Сложностные характеристики генетических текстов. В кн.: Труды 4-й Всероссийской конф. "Распознавание образов и анализ изображений», ч. 1. 1998, Новосибирск, с. 83-87.

12. Гусев В. Д., Немытикова Л. А., Учет проявлений повторности, симметрии и изоморфизма в символьных последовательностях: алгоритмические аспекты. Вычислительные системы (2001) 167, 11-33.

13. Зубков А. М., Неравенства для вероятностей переходов с запрешениями и их применения. Матем. сб. (1979) 109 (151), №4 (8), 491-532.

14. Зубков А. М., Оценки для сумм конечно-зависимых индикаторов и для момента первого наступления редкого события. Труды МИАН (1986) 177, 33-46.

15. Зубков А. М., Михайлов В. Г., Предельные распределения случайных величин, связанных с длинными повторениями в последовательности независимых испытаний. Теория вероятностей и ее применения (1974) 19, №1, 173-181.

16. Зубков А. М., Михайлов В. Г., Оценка точности пуассоновской аппроксимации в задаче о размещении частиц по ячейкам. Теория вероятностей и ее применения (1978) 23, №4, 819-824.

17. Зубков А. М., Михайлов В. Г., О повторениях $s$-цепочек в последовательности независимых величин. Теория вероятностей и ее применения (1979) 24, №2, 267-279.

18. Зубков А. М., Попов Н. Н., Отношение частичного порядка, порожденное распределениями числа занятых ячеек. Матем. заметки (1982) 32, №1, 97-102.

19. Колчин В. Ф., Чистяков В. П., Предельные распределения числа непоявившихся $s$-цепочек в полиномиальной схеме. Теория вероятностей и ее применения (1974) 19, №4, 855-864.

20. Медведев Ю. И., Некоторые теоремы об асимптотическом распределении статистики $\chi^{2}$. Докл. AH CCCP (1970) 192, №5, 987-989.

21. Михайлов В. Г., Предельные распределения случайных величин, связанных с многократными длинными повторениями в последовательности независимых испытаний. Теория вероятностей и ее применения (1974) 19, №1, 182-187.

22. Михайлов В. Г., Центральная предельная теорема для числа неполных длинных повторений. Теория вероятностей и ее применения (1975) 20, №4, 880-884.

23. Михайлов В. Г., Центральная предельная теорема для неоднородных $U$-статистик от конечно зависимых случайных величин. Матем. сб. (1975) 98 (140), №4 (12), 624-634.

24. Михайлов В. Г., Асимптотическая нормальность в схеме конечно-зависимого размещения частиц по ячейкам. Матем. сб. (1982) 119, №4, 509-520.

25. Михайлов В. Г., Асимптотическая нормальность разделимых статистик от частот $m$-цепочек. Дискретная математика (1989) 1, №4, 92-103.

26. Михайлов В. Г., Явные оценки в предельных теоремах для сумм случайных индикаторов. Обозрение прикладной и промышленной математики (1994) 1, №4, 580-617. 
27. Михайлов В. Г., Оценка точности сложной пуассоновской аппроксимации по методу СтейнаЧена. Обозрение прикладной и промышленной математики (1996) 3, №4, 530-548.

28. Михайлов В. Г., Некоторые неравенства для функции распределения числа непоявившихся s-цепочек. Труды по дискретной математике (1997) 1, 221-226.

29. Михайлов В. Г., Оценка точности сложной пуассоновской аппроксимации для распределения числа совпадающих цепочек. Теория вероятностей и ее применения (2001) 46, №4, 713-723.

30. Михайлов В. Г., Неравенства для среднего числа повторений $m$-цепочек и для среднего числа непоявившихся $m$-цепочек из заданного класса. Труды по дискретной математике (2000) 3, 147-154.

31. Михайлов В. Г., Об асимптотических свойствах распределения числа пар $H$-связанных цепочек. Дискретная математика (2002) 14, №3, 122-129.

32. Тимашёв А. Н., О вероятности совпадения векторов частот исходов независимых полиномиальных схем. В кн.: Третья Всероссийская школа-коллоквиум по стохастическим методам. Тезисы докладов. ТВП, Москва, 1996, 156-157.

33. Тихомирова М. И., Об асимптотической нормальности числа непоявившихся $s$-цепочек. Дискретная математика (1992) 4, №2, 122-129.

34. Тихомирова М. И., Чистяков В. П., Об асимптотике моментов числа непоявившихся $s$-цепочек. Дискретиая математика (1997) 9, №1, 12-29.

35. Тихомирова М. И., Чистяков В. П., О статистических критериях отсутствующих $s$-грамм. Tpyды по дискретной математике (1997) 1, 265-278.

36. Шойтов А. М., Предельные распределения числа наборов $H$-эквивалентных отрезков в равновероятной полиномиальной схеме серий. Дискретная математика (2002) 14, №1, 82-98.

37. Шойтов А. М., Повторения значений функции от отрезков последовательности независимых испытаний. Дискретиая математика (2000) 12, №3, 49-59.

38. Шойтов А. М., Предельные распределения числа наборов $\boldsymbol{H}$-эквивалентных отрезков в последовательности независимых испытаний. Обозрение прикладной и промышленной математики (1999) 6, №1, 218-220.

39. Шойтов А. М., Связь отрезков отношением $H$-эквивалентности в схеме серий. Обозрение прикладюой и промышленюой математики (1999) 6, №1, 220-221.

40. Шойтов А. М., Верхняя оценка расстояния по вариации между распределением случайной величины, равной числу наборов $H$-эквивалентных отрезков в равновероятной схеме серий, и распределением Пуассона. Обозрение прикладиой и промынлениой математики (2000) 7 , №2, 546-547.

41. Шойтов А. М. О скорости сходимости числа наборов $\boldsymbol{H}$-эквивалентных отрезков в равновероятной схеме серий к сложному распределению Пуассона. Обозрение прикладной и промышленной математики (2001) 8, №1, 381-381.

42. Шойтов А. М., Сложное распределение Пуассона числа наборов $H$-эквивалентных отрезков в последовательности независимых испытаний. Обозрение прикладной и промышленной математики (2001) 8, №2, 816-816.

43. Arratia R., Gordon L., Waterman M. S., An extreme value theory for sequence matching. Ann. Statist. (1986) 14, №3, 971-993.

44. Arratia R., Waterman M. S., Critical phenomena in sequence matching. Ann. Probab. (1985) 13, №4, 1236-1249.

45. Arratia R., Gordon L., Waterman M. S., The Erdős-Rényi law in distribution, for coin tossing and sequence matching. Ann. Statist. (1990) 18, №2, 539-570.

46. Barbour A. D., Holst L., Janson S., Poisson approximation. Oxford University Press, Oxford, 1992.

2 Дискретная математика, т.15 №4 
47. Benczur A., Katai I., On the number of occurrences of sequence patterns. Acta Math. Hung. (1986) 47, №3-4, 371-382.

48. Bıggins J. D., A note on repeated sequences in Markov chains. Adv. Appl. Probab. (1987) 19, №3, 739-742.

49. Chrysaphinou O., Papastavridis S., A limit theorem on the number of overlapping appearances of a pattern in a sequence of independent trials. Probab. Theory Relat. Fields (1988) 79, 129-143.

50. Chrysaphinou O., Papastavridis S., A limit theorem on the number of non-overlapping occurrences of a pattern in a sequence of independent trials. J. Appl. Probab. (1988) 25, №2, 428-431.

51. Chrysaphinou O., Papastavridis S., The occurrence of sequence patterns in repeated dependent experiments. Теория вероятностей и ее применения (1990) 35, №1, 167-173.

52. Dembo A., Karlin S., Zeitouni O., Critical phenomena for sequence matching with scoring. Ann. Probab. (1994) 22, №4, 1993-2021.

53. Dembo A., Karlin S., Zeitouni O., Limit distribution of maximal non-aligned two-sequence segmental score. Ann. Probab. (1994) 22, №4, 2022-2039.

54. Gordon L., Schilling M. F., Waterman M., An extreme value theory for sequence matching. Probab. Theory Relat. Fields (1986) 72, 279-287.

55. Karlin S., Ost F., Count of long aligned word matches among random letter sequences. Adv. Appl. Probab. (1987) 19, №2, 293-351.

56. Karlin S., Ost F., Maximal length of common words among random letter sequences. Ann. Probab. (1988) 16, №3, 535-563.

57. Karnin E. D., The first repetition of a pattern in a symmetric Bernoulli sequence. J. Appl. Probab. (1983) 20, 413-418.

58. Lempel A., Ziv J., On the complexity of finite sequences. IEEE Trans. Inf. Theory (1976) 22, №1, $75-81$.

59. Rukhin A. L., Distribution of the number of words with a prescribed frequency and tests of randomness. Adv. Appl. Prob. (2002) 34, 775-797.

Статья поступила 15.09.2003. 